\title{
Peripheral and central hyperexcitability: Differential signs and symptoms in persistent pain
}

\author{
Terence J. Coderrea \\ aPain Mechanisms Laboratory, Clinical Research Institute of Montreal; \\ Centre de recherches en sciences neurologiques et département de \\ médecine, Université de Montréal; Department of Psychology, McGill \\ University, Montreal, Quebec, Canada, H2W IR7 \\ Electronic mail: coderrt@ircm.umontreal.ca \\ Joel Katzb \\ Department of Psychology and Acute Pain Research Unit, The Toronto \\ Hospital; Departments of Behavioral Science and Anaesthesia, University of \\ Toronto, Toronto, Ontario, Canada M5S 2C4 \\ Electronic mail: j.katz@utoronto.ca
}

\begin{abstract}
This target article examines the clinical and experimental evidence for a role of peripheral and central hyperexcitability in persistent pain in four key areas: cutaneous hyperalgesia, referred pain, neuropathic pain, and postoperative pain. Each suggests that persistent pain depends not only on central sensitization, but also on inputs from damaged peripheral tissue. It is instructive to think of central sensitization as comprised of both an initial central sensitization and an ongoing central sensitization driven by inputs from peripheral sources. Each of these factors, initial sensitization, ongoing central sensitization, and inputs from peripheral sources, contributes to the net activity in dorsal horn neurons and thus influences the expression of persistent pain or hyperalgesia. Since each factor, peripheral inputs and central sensitization (initial or ongoing), can contribute to both the initiation and maintenance of persistent pain, therapies should target both peripheral and central sources of pathology.
\end{abstract}

Keywords: hyperalgesia; neurogenic inflammation; neuropathic pain; nociception; phantom limb pain; plasticity; postoperative pain; pre-emptive analgesia; referred pain; sensitization

\section{Introduction}

Pain is a normal reaction of the somatosensory system to noxious stimulation which alerts the individual to actual or potential tissue damage. It serves a protective function, informing us of injury or disease, and usually remits when healing is complete or the condition is cured. However, in some cases, peripheral tissue damage or nerve injury leads to a pathological state characterized by one or more of the following: pain in the absence of a noxious stimulus (spontaneous pain), increased duration of response to brief stimulation (ongoing pain or hyperpathia), reduced pain threshold (allodynia), increased responsiveness to suprathreshold stimulation (hyperalgesia), and spread of pain and hyperalgesia to uninjured tissue (referred pain and secondary hyperalgesia). For more than a century there has been a heated debate over the role of peripheral and central neural mechanisms in the initiation and maintenance of these pathological conditions. Although the debate has a long history (see Bonica 1992 for a review), most of the empirical evidence in support of one side or the other is relatively new (also see Ruda \& Dubner 1992; Willis 1994; Woolf 1992). Perhaps even newer is a growing realization that peripheral and central neural mechanisms are not mutually exclusive, and interact extensively to reinforce the pathological changes that contribute to chronic pain. In this paper we examine both clinical and experimental evidence for peripheral and central neural contributions to pathological pain. In this context, we will review the current state of knowledge concerning the proposed neural mechanisms contributing to the initiation and maintenance of four types of painful conditions, including: (1) hyperalgesia after cutaneous injury; (2) referred pain and hyperalgesia after deep tissue injury; (3) neuropathic pain; and (4) postoperative pain.

\section{Cutaneous hyperalgesia}

After a cutaneous injury both the injured skin and the uninjured skin adjacent to the injury become more sensitive to specific types of sensory stimulation. In particular, the injured skin becomes more sensitive to non-noxious heating or stroking (thermal and mechanical allodynia), as well as to noxious heating or punctate stimulation (thermal and mechanical hyperalgesia). In contrast to the injured skin, the 
adjacent uninjured skin appears to become more sensitive to mechanical, but not thermal stimuli, with hyperalgesia to punctate stimulation spreading further and lasting much longer (13-24 hrs) than allodynia to stroking (1-2 hrs) (Meyer et al. 1994). For descriptive purposes, many investigators have adopted the terminology first proposed by Hardy et al. (1950) in which hyperalgesia at the site of injury is termed primary hyperalgesia, and hyperalgesia in the adjacent uninjured skin is termed secondary hyperalgesia. Given a general prevalence of mechanical hyperalgesia over thermal hyperalgesia in this review, subsequent usage of the term hyperalgesia refers to mechanical hyperalgesia, unless otherwise specified.

2.1. Nociceptor sensitization. Most would agree that the thermal allodynia and hyperalgesia present in the injured region is due to peripheral sensitization of nociceptors. Following tissue injury there is an increase in the excitability of primary afferent nociceptors. Nociceptor sensitization is reflected by one or more of the following: decreased threshold, increased impulse frequency to the same stimulus, decreased latency of the first impulse, after discharge following extended or intense stimulation, and the appearance of spontaneous firing (Beitel \& Dubner 1976). Repeated heat stimulation produces nociceptor sensitization which develops within 1 minute and last for hours (Perl 1976). Following heating of the skin, sensitization to further heat stimuli has been demonstrated in C-fiber polymodal nociceptors in the rat (Lynn \& Carpenter 1982), rabbit (Perl et al. 1974), cat (Bessou \& Perl 1969), monkey (Beitel \& Dubner 1976) and man (Torebjörk et al. 1984). Sensitization after heat injury has also been found in the heat responses of $\mathrm{A}$-delta fiber, high-threshold mechanoreceptor units in the rabbit and cat (Fitzgerald \& Lynn 1977) and monkeys (Meyer \& Campbell 1981), as well as in the paradoxical responses of cold receptors in monkeys (Dubner et al. 1975).

Whether nociceptor sensitization can account for primary mechanical hyperalgesia is less clear. Thus, some investigators have found that both polymodal nociceptors (Bessou \& Perl 1969) and high threshold mechanoreceptors (Fitzgerald \& Lynn 1977) become sensitized to mechanical stimuli following heat injury. However, others have found that, within their normal receptive fields, the thresholds of C- and A-fiber mechanoheat sensitive nociceptors are not altered by heat or mechanical injury (J. N. Campbell et al. 1988a; Thalhammer \& LaMotte 1982). It has been alternatively suggested that the expansion of receptive fields of nociceptors into an adjacent area of injury may account for primary hyperalgesia to mechanical stimulation, as this occurs after heat (Thalhammer \& LaMotte 1982) and mechanical (Reeh et al. 1987) injuries.

Recent studies have looked for a correlation between nociceptor sensitization and reports of primary hyperalgesia following a cutaneous injury. Initially this was performed by comparing magnitude estimations of hyperalgesia in man with neurophysiological recordings in nerve fibers of monkeys (LaMotte et al. 1982; Meyer \& Campbell 1981). More recent studies have examined the correlation between human sensory judgements and evoked neural responses in the same subjects using percutaneous recording techniques (Ochoa \& Torebjörk 1989; Torebjörk et al. 1984). The results of these studies have been controversial. While Meyer and Campbell (1981) reported that primary hyperalgesia is associated with a sensitization of A-fibers and a desensitization of C-fibers, LaMotte et al. (1982) and Torebjörk et al. (1984) suggested that primary hyperalgesia is related to a sensitization of $\mathrm{C}$-fibers and not $\mathrm{A}$-fibers. It has been proposed that this discrepancy depends on either the type of skin that is injured or the intensity of the stimulus producing the injury.

2.2. Peripheral neurogenic mechanisms. The spread of hyperalgesia to uninjured tissue is probably not due to nociceptor sensitization, but may involve either a neurogenic axon reflex or a sensitization of central neurons. Lewis $(1936 ; 1937)$ was the first to perform an extensive examination of the spread of cutaneous hyperalgesia into uninjured tissue. According to Lewis, the spread of hyperalgesia to uninjured tissue was due to a peripheral neural mechanism which involved antidromic activity in peripheral nerves leading to the release of a substance which contributed to the development of both hyperalgesia and vasodilatation or flare responses in the skin. In support of his hypothesis, Lewis presented evidence that cutaneous hyperalgesia in response to skin crush does not develop in anesthetized skin and does not spread across an anesthetized strip of skin, until after the anesthesia wears off. Lewis also showed that cutaneous hyperalgesia which occurred in response to electrical stimulation of nerves through the skin (faradic stimulation), was prevented by a local anesthetic nerve block distal to the electrical stimulus. Conversely, when the nerve block was proximal to the faradic stimulation, hyperalgesia developed normally, but only after the anesthesia wore off.

Early studies provided supported for Lewis's mechanism of spreading hyperalgesia. Perl et al. (1974) showed that an extensive skin injury produced a sensitization of C-fiber polymodal units whose receptive fields were removed from the injured region. Fitzgerald (1979) recorded activity in C-fiber nociceptors in the skin near an injury and found that nociceptors in the uninjured tissue were more sensitive to heat following an injury than when there was no injury. The spread of the effect of the injury was induced by nerve impulses, since a local injection of lignocaine anesthetic blocked the spread of sensitization. Fitzgerald also found that there was a spread of nociceptor sensitization following antidromic stimulation of the rabbit sural nerve at C-fiber strength. The effect was independent of the CNS (central nervous system) since it occurred even when the nerve was cut central to the stimulation point. Chahl and Ladd (1976) demonstrated that antidromic stimulation of the rat saphenous nerve produced inflammation and an increased excitability in sensory nerve fibers when the stimulation was of C-fiber, but not A-fiber strength.

More recent studies provide evidence against Lewis's theory of spreading peripheral sensitization. Thus, antidromic stimulation of nociceptive fibers in either the monkey (Meyer et al. 1988) or the rat (Reeh et al. 1986) was not found to produce nociceptor sensitization. In addition, Thalhammer and LaMotte (1982) found that a heat injury in one half of a cutaneous nociceptor's receptive field did not produce heat sensitization in the other half, despite the fact that hyperalgesia spread into this area. Indeed, mechanical and chemical injuries produce extensive, spreading hyperalgesia (LaMotte et al. 1992) without producing the same degree of spreading sensitization of primary afferent nociceptors in monkeys ( J. N. Campbell et al. 
1988a; Baumann et al. 1991) or humans (LaMotte et al. 1992). Typically, nociceptor sensitization associated with injury is restricted to about $5-10 \mathrm{~mm}$ of the site of injury (J. N. Campbell et al. 1984; Fitzgerald 1979), while cutaneous hyperalgesia spreads as far as $10-20 \mathrm{~cm}$ beyond the site of injury (Hardy et al. 1950; LaMotte et al. 1991; 1992; Lewis 1936; 1937). Furthermore, the zone of secondary hyperalgesia is typically found to be larger than the flare produced by tissue injury (Koltzenburg et al. 1992; LaMotte et al. 1991; Raja et al. 1984). In fact, as noted by LaMotte et al. (1991), a flare can be produced (by histamine injection) without even inducing secondary hyperalgesia, and secondary hyperalgesia can occur in the absence of a flare response. Finally, secondary hyperalgesia after cutaneous injury typically does not spread beyond the body's midline, whereas flare responses do (LaMotte et al. 1991).

2.3. Central sensitization. In contrast to Lewis, Hardy et al. (1950) proposed that while primary hyperalgesia was mediated by peripheral mechanisms, secondary hyperalgesia was produced by central sensitization. Hardy et al. (1950) confirmed Lewis's finding that cutaneous hyperalgesia (in this case in response to burn injury) did not develop in anesthetized skin until after the anesthesia wore off. However, in contrast to Lewis, Hardy et al. reported that hyperalgesia after faradic stimulation was unaffected by a distal nerve block, but was significantly delayed by a proximal nerve block.

More recent evidence supports the view that hyperalgesia depends, in part, on central sensitization (Guilbaud et al. 1992b; LaMotte 1992; Torebjörk 1992). Hyperalgesia to punctate mechanical stimuli, which develops after intradermal injection of capsaicin, is maintained even after anesthetizing the region where capsaicin was injected (LaMotte et al. 1991). However, if the skin region is anesthetized prior to capsaicin injection, cutaneous hyperalgesia does not develop. Furthermore, hyperalgesic responses to capsaicin can be prevented if the area of skin where the injection is made is rendered anesthetic by a proximal anesthetic block of the peripheral nerve which innervates it. Thus, for hyperalgesia to develop it is critical that initial inputs from the injury reach the CNS. However, once hyperalgesia is established, it does not need to be maintained by inputs from the injured peripheral tissue. In support of this, Torebjörk et al. (1992) have shown that pain thresholds to intraneural electrical stimulation of afferent fibers are dramatically reduced following intradermal capsaicin injection in the skin from which the stimulated nerve emanates: neural stimulation which was felt as tactile before administration of capsaicin, was painful after capsaicin. Importantly, this reduced pain threshold is evident even when the sensory projected field of the afferent nerve is anesthetized after the capsaicin injection. Again, a state of central sensitization is indicated since once they have established their effects, inputs from the injured region are not required to maintain the lowered threshold.

Recent experimental data from animal studies also provide support for Hardy et al.'s central mechanism of secondary hyperalgesia since peripheral injuries typically produce a sensitization of neurons in central nervous system (CNS). Thus, dorsal horn neurons fire with increasing frequency in response to repeated application of a noxious heat stimulus (Kenshalo et al. 1979; Perl 1976). Sensitization of dorsal horn neurons occurs after various types of tissue damage including thermal injury (Kenshalo et al. 1982; Price et al. 1978), chemical injury (Dougherty \& Willis 1992; Simone et al. 1991), and polyarthritis (Calvino et al. 1987; Menétrey \& Besson 1982), or after stimulation of C-fiber afferents (Chung et al. 1979). Tissue injury/inflammation or electrical nerve stimulation also produces sensitization in spinal motoneurons (Woolf 1983), thalamus (Guilbaud et al. 1986), and somatosensory cortex (Lamour et al. 1983). Repeated C-fiber afferent stimulation also produces a sequential increase in dorsal horn activity resulting in a prolonged discharge of the cell (wind-up), which lasts from seconds to minutes post-stimulation (Mendell 1966; Schouenbourg \& Dickenson 1985).

In addition to the sensitization and wind-up of dorsal horn cells, noxious stimulation associated with tissue injury also produces an expansion of the receptive fields of dorsal horn neurons. Neurons in the dorsal horn of the spinal cord with receptive fields adjacent to a cutaneous heat injury expand their receptive fields to incorporate the site of injury (McMahon \& Wall 1984). Similar receptive field expansions have been observed in spinal cord following mechanical (Cervero et al. 1988), chemical (Hoheisel \& Mense 1989; Woolf \& King 1990), and inflammatory (Hylden et al. 1989) injuries, as well as following the induction of polyarthritis (Calvino et al. 1987; Menétry \& Besson 1982), and in response to electrical nerve stimulation (Cook et al. 1987). Inflammatory lesions also produce an expansion of receptive fields of cells in the ventrobasal thalamus (Guilbaud et al. 1986). Injury-induced receptive field expansions may contribute to enhanced pain by recruiting primary afferent fibers within the newly expanded field, thus increasing the magnitude of the ascending signal into the CNS, or by modality convergence and activation of previously ineffective synapses (Devor 1989; Dubner et al. 1987). It should be noted, however, that the degree of expansion of neuronal receptive fields is not necessarily related to pain sensation, and varies greatly with the level of anesthesia.

Behavioral and physiological studies in animals also demonstrate hyperalgesia or an increase in the excitability of flexor efferent responses to stimulation of body regions which are at a distance from a cutaneous or deep tissue injury. Woolf (1984) found that localized thermal and chemical injuries cause reductions in flexion reflex thresholds to noxious mechanical and thermal stimulation in the limb contralateral as well as ipsilateral to the injury. Cutaneous (Woolf 1983) and deep (Woolf \& McMahon 1985) tissue injury, as well as noxious electrical stimulation of cutaneous and muscle afferent nerves (Wall \& Woolf 1984) also produce an increase in the excitability of the ipsilateral and contralateral flexor efferent nerves in response to noxious mechanical stimulation of the hindpaw. Since the increased excitability in the contralateral flexor efferent nerve is maintained even after inputs from the injured paw are blocked by local anesthesia, the results suggest that central, not peripheral, changes underlie this effect. In this way, cutaneous hyperalgesia may depend on central sensitization which is produced by inputs from a peripheral injury, but does not need to be maintained by them. Behavioral studies of thermal withdrawal latencies indicate that the spread of hyperalgesia to the hindpaw contralateral to the paw that received a thermal injury is unaffected by either deafferentation or anesthetic blocks of the injured hindpaw following the injury, but is prevented if deafferen- 
tation or anesthetic block precedes the injury (Coderre \& Melzack 1985; 1987). The similarity between the physiological and the behavioral data is quite striking considering that the flexor efferent reflex measures were obtained in decerebrate/spinalized animals, while withdrawal latencies were obtained in intact, awake animals. These data provide further evidence that peripheral injury can produce central changes which are maintained even after the inputs from the injury are removed.

\subsection{Separate contribution of peripheral and central sensi-} tization to hyperalgesia. The great debate between Lewis and Hardy et al. began chiefly because of their very different interpretations of similar results they each obtained from experiments comparing the effects of proximal and distal nerve blocks on the development of spreading hyperalgesia after faradic stimulation of the skin over peripheral nerves. Lewis concluded that spreading hyperalgesia was mediated by a peripheral neural mechanism because the hyperalgesia was blocked by distal but not proximal nerve blocks. Hardy et al. concluded that secondary hyperalgesia was mediated by a central neural mechanism because the hyperalgesia was unaffected by distal nerve blocks, but was delayed by proximal nerve blocks. While the conclusions based on their apparently different findings were opposite, in reality their experimental findings were very similar (see Fig. 1). First, both Lewis and Hardy et al. found that hyperalgesia to faradic stimulation develops fully after proximal nerve blocks wear off. Lewis reported that hyperalgesia extended throughout the anesthetized region after the nerve block wore off (between 15 and $60 \mathrm{~min}$ after stimulation) (Fig. 1B). In Hardy et al.'s experiment, hyperalgesia extended throughout the anesthetized region by 60 min after stimulation (Fig. 1A). Thus, the delay in the spread of hyperalgesia in Hardy et al.'s study was not greater than the variability in the length of anesthesia reported by Lewis.

In the case of distal nerve blocks, there is again no real difference in their data. Although Lewis concluded that hyperalgesia does not spread into the anesthetized region, it is evident from his figures (Lewis 1936, Fig. 6, or Fig. 1D here) that in some cases hyperalgesia did spread up to $2 \mathrm{~cm}$ into the anesthetized region. Hardy et al. concluded that hyperalgesia does spread into the anesthetized region after distal nerve blocks. However, when Hardy et al. stimulated nerve trunks at the same distance $(3 \mathrm{~cm})$ from the distal nerve block as did Lewis, they also found that hyperalgesia spread only $2-3 \mathrm{~cm}$ into the anesthetized region (Hardy et al. 1950, Fig. 8B or Fig. 1C here). Given all the variability inherent in assessing the borders of secondary hyperalgesia, performing nerve blocks, and stimulating nerve trunks through the skin, as well as the normal variability in the development of hyperalgesia seen between different subjects receiving the same stimulus, the similarities of their findings are more impressive than the differences. Thus, it appears that Lewis and Hardy et al. came to completely opposite conclusions with nearly identical data.

What conclusion do we come to from Lewis's and Hardy et al.'s data? With distal nerve blocks, Hardy et al., like Lewis, found very little spread of hyperalgesia into the anesthetized zone, suggesting that peripheral neural mechanisms are critical to secondary hyperalgesia. As for proximal blocks, the fact that both Lewis and Hardy et al. found that the full extent of hyperalgesia to faradic stimulation
A
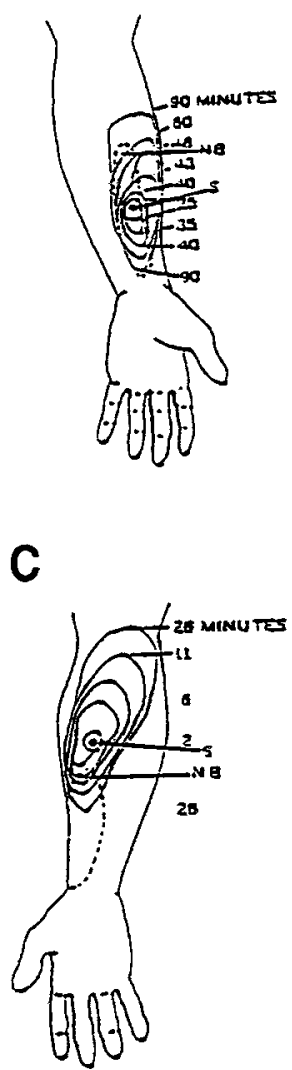

B

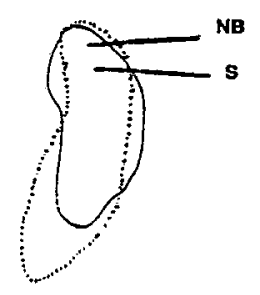

D

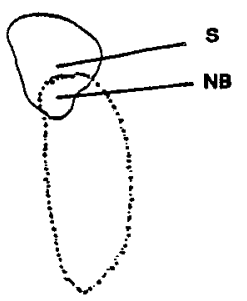

Figure 1. Similarities in the effects of proximal (A \& B) and distal (C \& D) nerve blocks on hyperalgesia produced by faradic stimulation in studies by Hardy et al. (1950; left side) and Lewis (1936; right side). Nerve blocks (NB) and stimulation (S) were performed where indicated; zones of anesthetic skin and hyperalgesia are indicated by dashed and solid lines, respectively. Lewis's diagrams have been flipped vertically, reduced in scale, and relabeled to enable an easier comparison with those of Hardy et al. Both Hardy et al. (A) and Lewis (B) found extensive spread of hyperalgesia throughout the previously anesthetic zone within 60 min of a proximal nerve block. In contrast, both Hardy et al. (C) and Lewis (D) observed a small, but similar, degree of hyperalgesia spreading into the anesthetic zone after a distal nerve block. Modified from Hardy et al. (1950) and Lewis (1936), with permission.

develops after the block wears off, could be taken as evidence, as Lewis suggests, that central neural mechanisms do not contribute to secondary hyperalgesia. Although the proximal nerve block would prevent neural impulses from reaching the central nervous system during the faradic stimulation, it is highly likely that the intense electrical stimulation required to penetrate the skin and activate high threshold nerve fibers would produce considerable tissue injury (including that produced by peripheral neurogenic and non-neurogenic proceses). Consequently, it is also possible that peripheral tissue injury associated with the faradic stimulation could, after the proximal nerve block wears off, produce central neural changes which contribute to the development of secondary hyperalgesia.

There is no doubt that the faradic stimulation used by Lewis and Hardy et al. did produce extensive tissue injury. In many experiments the skin was stimulated for $5 \mathrm{~min}$ with 
a current at the pain tolerance level. Furthermore, when the skin was infiltrated with a local anesthetic prior to electrical stimulation, there was a delay in the development of secondary hyperalgesia until after the anesthesia wore off. Since the skin was anesthetized during the stimulation, thus preventing axon reflexes and central transmission, it is clear that the electrical stimulation itself did not induce the hyperalgesia. Recently, Dahl et al. (1993) have shown that there is a similar delay in the development of secondary hyperalgesia in response to burn injury $\left(50^{\circ} \mathrm{C}\right.$ for $\left.6 \mathrm{~min}\right)$ performed within an anesthetized patch of skin. Thus, it appears that an injury performed in anesthetized skin will produce hyperalgesia which appears after the anesthesia has waned, provided the injury is capable of producing nonneurogenic tissue injury.

In contrast to the effects of faradic stimulation or burn injury, the hyperalgesia produced by capsaicin probably involves minor non-neurogenic tissue injury. Subcutaneous injection of capsaicin produces a small bleb at the injection site, which is no larger than that produced by its vehicle, and typically does not even produce a weal (LaMotte et al. 1991). Furthermore, when capsaicin is injected into anesthetized skin, unlike the effects of electrical and burn injuries of the skin, hyperalgesia does not develop at all (LaMotte et al. 1991). For this reason, we suggest that capsaicin is a better stimulus to use when one wishes to assess the effects of brief sensitization, independent of lingering peripheral inputs. Importantly, it has also been found that the secondary hyperalgesia which occurs following burn injury typically does not last as long as the primary hyperalgesia at the site of injury. Moinche et al. (1993) reported that primary hyperalgesia after heat injury persisted for 48 hours, while secondary hyperalgesia to punctate mechanical stimuli did not extend beyond 24 hours. In contrast, LaMotte et al. (1991) found that localized thermal hyperalgesia after capsaicin injury persisted for only 1-2 hrs, while secondary hyperalgesia to punctate mechanical stimuli lasted 13-24 hrs.

It is significant that of the four stimuli that commonly have been used to produce secondary hyperalgesia (faradic stimulation, skin crush, burn, and capsaicin injection), it is only after capsaicin injection that secondary hyperalgesia is prevented by prior proximal nerve block or local anesthesia of the skin; the other stimuli invariably lead to a full blown secondary hyperalgesia, which is delayed until the anesthetic wears off. Based on these findings, we hypothesize that prior anesthetic nerve or skin blocks will prevent subsequent hyperalgesia only if there is minimal tissue damage and no continued activation of primary afferents. If this is true, then it would be very difficult to find support for a contribution of central neural mechanisms of hyperalgesia by assessing the effects of prior anesthetic blocks with stimuli which produce extensive tissue injury or continued afferent input.

Since peripheral injury interferes with the ability to assess the contribution of central neural mechanisms of hyperalgesia using prior anesthetic blocks, perhaps more useful information can be gained using postinjury blocks. Lewis (1936) found that hyperalgesia was completely unaffected by local anesthesia of the skin previously subjected to faradic stimulation. Hardy et al. (1950) found the hyperalgesia produced by faradic stimulation could be blocked by subsequent local anesthesia, but only with deep, as opposed to superficial, anesthetization. Dahl et al. (1993) demon- strated that postinjury local anesthesia of a burned region reduced, but did not eliminate, cutaneous hyperalgesia. In the case of capsaicin, LaMotte et al. (1991) found that hyperalgesia to stroking, but not the hyperalgesia to punctate stimuli, was blocked by local anesthesia or cooling of the skin at the site of injury, after hyperalgesia has fully developed. Thus, postinjury blocks have been found either to not affect, to completely block or to partially block hyperalgesia associated with various stimuli.

\subsection{Contribution of initial and ongoing central sensitiza-} tion to hyperalgesia. From the above discussion of cutaneous hyperalgesia it appears that both peripheral and central neural mechanisms may contribute to secondary hyperalgesia, but that any determination of their separate roles is highly controversial. From the above two paragraphs we recognize that the ability to demonstrate a central contribution to secondary hyperalgesia is very much dependent on the degree of tissue injury produced by the stimulus. However, it is likely that both peripheral and central sensitization become more obvious with a greater degree of peripheral injury. We propose that rather than emphasizing the separate roles of peripheral and central neural mechanisms to cutaneous hyperalgesia, the existing data can be better explained by a hypothesis that involves an interactive contribution of both peripheral and central sensitization (see Fig, 2). In addition to this basic interaction, it is important to conceive of central sensitization as composed of two components, initial central sensitization and ongoing central sensitization, which is influenced by peripheral sensitization.

We know that both a brief afferent barrage produced by C-fiber stimulation and the persistent inputs associated with peripheral tissue injury will each sensitize dorsal horn neurons. Thus, when studying the role of central sensitization in hyperalgesia, it is relevant to differentiate between the initial central sensitization produced by an injury barrage and the ongoing central sensitization associated with lasting tissue injury. In this way, the sensitized state can depend on either one of two processes: an initial intense barrage, or an ongoing lower-level peripheral input. Initial sensitization could be considered an autonomous state that exists without an ongoing or maintaining input, that is, the sensitization which persists after the initial stimulation. In contrast, ongoing sensitization is a more labile state that exists only if there is ongoing peripheral input to maintain it. The separation of central sensitization into these two components may explain many of the differences obtained in studies which assess the effects of pre- or postinjury local anesthesia blockade on secondary hyperalgesia. We hypothesize that with severe tissue injury the afferent input is so intense at the time of testing, that hyperalgesia will be evident regardless of whether or not preinjury anesthetic block was performed. Thus, ongoing central sensitization maintained by the inputs from damaged peripheral tissue overrides much of the benefit of the preinjury block. However, with less extensive tissue injury a preinjury block will reduce or prevent the initial central sensitization which would be produced by the injury barrage. Since there is little ongoing central sensitization associated with minor injury, the secondary hyperalgesia is prevented. Thus, preinjury anesthetic blocks are typically used to provide information about initial central sensitization; however, if there is sufficient ongoing central sensitization, as in the case of 


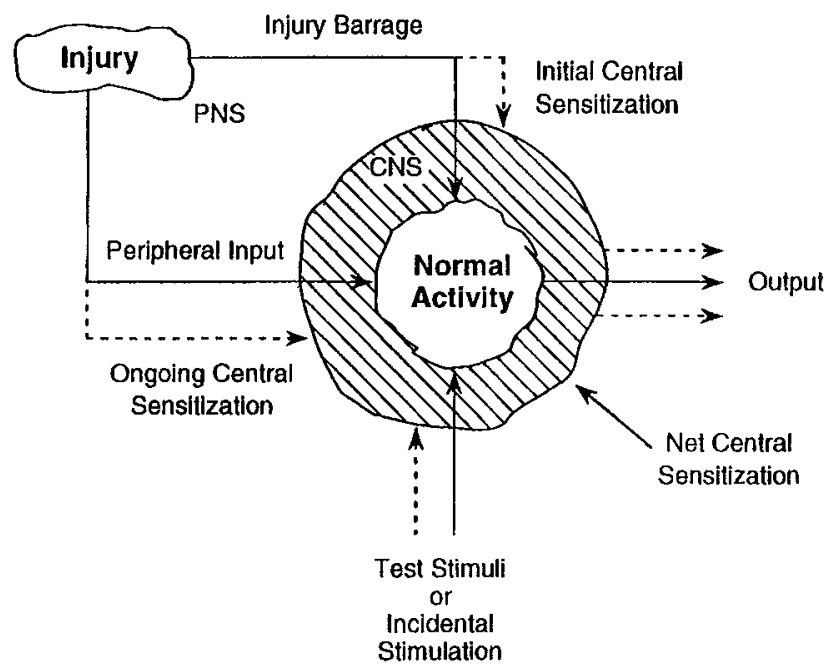

Figure 2. Schematic model of the proposed contribution of both initial and ongoing central sensitization to the output of central nervous system (CNS) neurons that are involved in the processing of nociceptive information. An injury produces both an injury barrage, which underlies initial sensitization, and continued peripheral inputs, which underlie ongoing sensitization of central nociceptive neurons. The combined influence of both initial and ongoing sensitization results in a significant decrease in the threshold of central neurons to further stimulation and is represented by the depiction of a larger area of activation for net central sensitization (diagonal hatched-line fill), as compared with normal activity (open fill). Pain sensitivity (hyperalgesia) and spontaneous pain are influenced by the output of the central nociceptive neurons. The output of central nociceptive neurons is directly influenced by the degree of net central sensitization and by the degree of peripheral inputs which activate afferent fibers that impinge on sensitized neurons. Importantly, peripheral inputs may originate from the injury itself, or from adjacent or remote uninjured regions whose afferent fibers converge directly (monosynaptically) or indirectly (polysynaptically) onto the sensitized neurons. In this way, the output of sensitized neurons reflects not only the degree of input from the injured region, but also the input from test stimuli in an area of secondary hyperalgesia, or incidental stimulation of referred zones (after visceral or deep tissue injury) or focal points (after nerve injury). The dashed-line arrows represent the enhanced output which is associated with an enhanced sensitivity to peripheral inputs, and an enhanced activation of the sensitized central neurons, as compared to the solid-line arrows which represent normal sensitivity, activation, and output of the central nociceptive neurons.

extensive tissue injury, information about initial sensitization is better obtained using postinjury anesthetic blocks in order to eliminate the confounding effects of ongoing central sensitization associated with the peripheral injury. In these cases, a role of initial central sensitization is suggested if hyperalgesia persists despite the block. However, hyperalgesia which is dependent on ongoing rather than initial central sensitization is reduced or eliminated by post-injury block.

We also propose that initial and ongoing central sensitization, although temporally separated, are interdependent. Thus, the degree of hyperalgesia is related to the net central sensitization, which derives both from initial and ongoing central sensitization. If the initial central sensitization is great enough, then hyperalgesia may be less dependent on ongoing peripheral inputs. If the ongoing central sensitiza- tion is great enough, then hyperalgesia is less dependent on the initial central sensitization. Accordingly, very severe injuries such as faradic stimulation, or burns, that elicit both an intense afferent barrage, as well as subsequent tissue injury, would produce hyperalgesia that involves both a strong initial central sensitization and significant ongoing central sensitization. Since the net central sensitization and resulting hyperalgesia reflect both initial and ongoing central sensitization, in such cases pre-injury anesthetic blocks would only delay hyperalgesia (i.e., it would develop later due to ongoing central sensitization). Similarly, by eliminating ongoing central sensitization, postinjury anesthetic blocks would either not affect, or only partially reduce, hyperalgesia, because the initial sensitization is sufficient to maintain a level of net central sensitization required to produce hyperalgesia.

In the case of a capsaicin injection, where the afferent barrage due to C-fiber activation is high, but the tissue injury is considerably less, the net central sensitization and hyperalgesia depend more on initial sensitization and less on ongoing central sensitization. Thus, preinjury anesthetic blocks are very effective because they reduce the initial central sensitization, but postinjury anesthetic blocks are less effective because the ongoing sensitization is less critical. In contrast, recent evidence (Koltzenburg et al. 1994) suggests that hyperalgesia produced by the topical application of mustard oil may depend more on ongoing central sensitization. Thus, it was established that after mustard oil treatment the degree of brush-evoked secondary hyperalgesia was highly correlated with the degree of ongoing burning pain from the site of injury.

In a recent article in the IASP Newsletter, Niv and Devor (1993) raised an important question about the time constant of central sensitization. It was suggested that since secondary stroking hyperalgesia produced by chemical irritation of the skin is diminished by cooling or anesthetizing the injured area, the time constant of central sensitization is short-lived. It was implied that because local anesthetic blockade temporarily relieved secondary hyperalgesia, the central sensitization that underlies the hyperalgesia was eliminated - at least until it was reinitiated after the blocks wore off. We would recommend caution in equating central sensitization and secondary hyperalgesia. While central sensitization may contribute to secondary hyperalgesia, the presence of secondary hyperalgesia does not automatically imply that central sensitization is present, and its absence does not necessarily imply that central sensitization has been eliminated. Importantly, peripheral inputs from chemically irritated skin will retrigger secondary hyperalgesia after a post-treatment anesthetic nerve block, but produce substantially reduced or no secondary hyperalgesia if the nerve block was present at the time of the chemical irritation (Koltzenburg et al. 1994; LaMotte et al. 1991). It is also important to distinguish the concepts of reinitiating versus retriggering of central sensitization. Reinitiating implies that the sensitization has disappeared and must be initiated once again by a similar peripheral input, while retriggering implies the central sensitization did not disappear, but was latent, and is re-established when a necessary threshold is met by peripheral inputs. As pointed out by Gracely et al. (1992), retriggering is more likely than reinitiation, since when first initiated secondary hyperalgesia and allodynia gradually expands outward from the injury, yet when returning after a local anesthetic block of 
the injured area, the hyperalgesia and allodynia rapidly expands over its previously existing area.

LaMotte at al.'s (1991) data with capsaicin not only point to the importance of the interaction of initial and ongoing central sensitization, but also attest to the importance of the inputs produced by the test stimulus, since postinjury local anesthetic blockade of the skin more effectively alleviated hyperalgesia to stroking than hyperalgesia to punctate stimuli. The fact that hyperalgesia to stroking is reduced by postinjury blockade, suggests that stroking hyperalgesia is maintained by ongoing central sensitization of the injury. In contrast, the fact that hyperalgesia to punctate stimuli is not alleviated by postinjury blocks, suggests that hyperalgesia to punctate stimuli is not maintained by ongoing central sensitization. We propose that since hyperalgesia ultimately depends on the net activity of dorsal horn neurons, the stroking stimulus may not provide enough input to produce pain when ongoing central sensitization has been abolished by the postinjury local anesthetic blockade. In contrast, the greater sensory input produced by pricking the skin will be sufficient to activate dorsal horn neurons, which have been sensitized by the capsaicin injury barrage (i.e., initial central sensitization), despite the elimination of ongoing central sensitization by the postinjury local anesthetic. In this way, ongoing central sensitization combines with initial sensitization to enhance incoming inputs which must meet or exceed a specific threshold before pain or hyperalgesia is experienced. The elimination of one of these components does not necessarily eliminate hyperalgesia if inputs from one of the other two components is strong enough. It is possible, however, that it is not exclusively sensory intensity that is important, but rather whether high or low threshold afferents are excited, so that high threshold input associated with punctate stimuli is able to overcome the effects of the anesthetic block, while low threshold input associated with stroking is not.

Two phenomena have not adequately been explained by the model in Figure 2: (1) Heat hyperalgesia does not spread into uninjured tissue to the same extent as mechanical hyperalgesia, and (2) mechanical hyperalgesia does not spread across an anesthetized strip of skin. To explain these phenomena LaMotte et al. (1991) proposed that, in addition to mechanoheat sensitive nociceptors and low threshold mechanoreceptors which have less extensive branching, there is a population of chemospecific afferent fibers which branch extensively in the skin; a theory which is now supported experimentally (Schmelz et al. 1994). According to LaMotte et al., capsaicin injury activates the chemospecific afferents which release a neuromodulator from their central terminals. This neuromodulator sensitizes dorsal horn neurons that receive input from myelinated low or high threshold mechanoreceptive afferents. These dorsal horn neurons in turn facilitate the responses of wide dynamic range neurons and high threshold spinothalamic tract (STT) neurons to mechanical stimulation of the skin outside the area of injury, while separate dorsal horn neurons facilitate STT neuron responses to heat stimulation inside the area of injury. According to LaMotte et al., this explains why there is remote hyperalgesia to mechanical but not heat stimuli. LaMotte's model is also proposed to explain the effects of a local anesthetic strip since the anesthesia blocks neural conduction in the lateral branches of the widely branching chemospecific neurons. Although LaMotte's model has been endorsed and expanded upon by some investigators (Meyer et al. 1992), others have been skeptical (Lynn 1992; Wall 1993). In particular, Wall (1993) has suggested that the anesthetic skin strip findings could be explained by central effects, rather than indicating there are widely branched chemospecific afferents. Wall (1993) proposed that a strip of local anesthetic applied to the skin produces a strip of spinal cord cells which are unable to refer to the periphery, and thus blocks the spread of central changes from the original focus to neighbouring cells. Other explanations attribute the spread of mechanical hyperalgesia, despite the absence of spreading heat hyperalgesia, to the sensitization of afferents which are initially insensitive to mechanical stimulation. These afferent fibers develop a sensitivity to mechanical stimulation after exposure to inflammatory mediators released in injured skin (Davis et al. 1993; Kress et al. 1992), as occurs for a group of mechanically insensitive neurons (so-called silent nociceptors) in the inflamed knee joint of rats (Schaible \& Schmidt 1988), as well as in skin sensitized by capsaicin or mustard oil in humans (Schmelz et al. 1994; Schmidt et al. 1995).

\section{Referred pain and hyperalgesia}

First described by Martyn (1864), referred pain is a condition in which pain is not localized within the injured region, but at an adjacent or distant site. Referred pains often occur following injury of deep tissue such as muscle, joints or viscera. Indeed, referred pain and hyperalgesia are often reported in the muscle and skin within the same spinal cord dermatome as injured organs, and are commonly used in the diagnosis of conditions such as appendicitis and angina pectoris. It has been shown that the distribution of referred pain increases with the intensity (Kellgren 1937; 1938; McLelland \& Goodell 1943; Vecchiet et al. 1993) and duration (McAuliffe et al. 1943; McLelland \& Goodell 1943) of the noxious stimulation from the injured deep tissue. Typically referred pain is restricted to the same spinal segment, however in some cases it has been found to extend great distances and beyond segmental boundaries (Lewis 1942; Livingston 1943). Along with referred pain there is often a development of tenderness in the referred area (i.e., referred hyperalgesia) (Head 1893; Procacci et al. 1986). Importantly, referred pain and hyperalgesia typically develop slowly after injury of deep tissues (Kellgren 1939), similar to the development of secondary hyperalgesia after cutaneous injury (Lewis 1936; Hardy et al. 1950).

3.1. Theories of referred pain. Although several theories have been advanced to account for referred pain, there is general agreement that referred pain depends on neural mechanisms, since local anesthesia of the injured region blocks its expression (Robertson et al. 1947; Vecchiet et al. 1993). Aside from this single point of agreement, the various theories of referred pain have little in common. One theory proposes that referred pain depends on impulses arising from the injured deep tissue region producing a sensitization of the referred area by means of an axon reflex mechanism (Penfield 1925). Another theory suggests that while referred hyperalgesia is dependent on an axon reflex mechanism, referred pain depends on the misinterpretation of inputs from an injured region whose axons also branch to the uninjured referred area (Sinclair et al. 1948). A third, the convergence-projection theory (Ruch 1947), suggests that axons from the injured and referred regions 
converge on the same cells in the spinal cord and there is a misinterpretation as to the source of the stimulation. A fourth theory suggests that impulses within axons from the injured region produce a facilitation of cells in the spinal cord at which axons from the referred area also terminate (MacKenzie 1893). This convergence-facilitation theory of referred pain was inspired by earlier suggestions of Sturge (1883) and Ross (1888) that referred pain depended on the development of a "commotion" or "irritable focus" in spinal cord neurons. Other hypotheses for referred pain contend that it is due to a summation of inputs from the injured and referred area within neurons of the brain, rather than the spinal cord (Cohen 1947; Theobald 1941). The major difference between these theories is their reliance on either a peripheral or a central mechanism. The peripheral mechanism is dependent on axon reflexes and peripheral sensitization, and the central mechanism is dependent on convergence in the central nervous system with or without central sensitization.

\subsection{Evidence supporting peripheral or central sensitiza-} tion in referred pain. There is some experimental support for the idea that referred pain may rely on an axon reflexlike response in branched afferent nerves, as proposed in the theories of Penfield (1925) and Sinclair et al. (1948). It has been shown that $18 \%$ of unmyelinated lumbar splanchnic nerve fibers can be activated by electrical stimulation of somatic nerves (Bahr et al. 1981). Mense et al. (1981) have also reported that there are sensory neurons with bifurcating axons which innervate both skin and muscle in the cat's tail. However, as indicated by McMahon (1994), it is generally agreed that these types of neurons are rare, if they exist at all. Furthermore, it is unlikely that this branched nerve mechanism could explain the delayed onset of referred pain after deep tissue injury, and it fails to explain pain which has been found to be referred to deafferented areas (Brown 1942; 1948; Kellgren 1938; Livingston 1943). As described in the section on cutaneous hyperalgesia, there is also a debate as to whether antidromic stimulation can produce a spreading sensitization of nociceptors.

There is considerably more evidence to support the idea that referred pain relies on a convergence of inputs at the spinal cord level, as proposed in the theories of Ruch (1947) and MacKenzie (1893). In an extensive review of the literature on the studies of visceral afferent activity Ness and Gebhart (1990) listed over 60 experiments demonstrating the presence of spinal neurons which receive both visceral and somatic input. The percentage of neurons which received convergent input from viscera and somatic inputs ranged from 6 to $100 \%$, but was above $90 \%$ in the vast majority of these experiments. While these studies provide significant support for convergence-projection mechanisms of referred pain, they do not adequately explain either the slow development of referred pain or the appearance of tenderness in the referred area. Furthermore, there are many examples in which referred pain is eliminated or significantly reduced by local anesthesia of the referred area (see below).

The slow development of referred pain and hyperalgesia and the reduction of referred pain and hyperalgesia by local anesthesia of the referred area provide more support for a convergence-facilitation theory, since the sensitization of central neurons would most likely take some time to develop, and the resultant sensations would rely on a summa- tion of inputs from the injured and referred areas, and not simply a misinterpretation of the origin of the inputs.

Furthermore, a role of central mechanisms in referred pain is suggested by the observation that phrenic nerve stimulation causes referred shoulder pain even after sectioning all cutaneous nerves from the painful region of the shoulder (Doran \& Ratcliffe 1954), and by the finding that the injection of hypertonic saline into intraspinous ligaments produces pain referred to a phantom arm (Harman 1948). If referred pain could be explained exclusively by convergence, then such pains would not provide clear evidence of central sensitization. However, evidence that referred pain is also, in part, dependent on CNS changes is provided by findings that referred pain and hyperalgesia spread to areas which do not share the same dermatome (Lewis 1942; Livingston 1943). For example, it has been shown that pain of cardiac origin is referred to sites as distant as the patient's ear (Brylin \& Hindfelt 1984). That pain and hyperalgesia can spread to areas far removed from the injured region implies that central changes and facilitation, as opposed to convergence, are involved in the spread of hyperalgesia.

Referred pain has also been found to spread specifically to sites of a previous injury. Henry and Montuschi (1978) described a case where the pain of an angina attack was referred to the site of an old vertebral fracture, while Cohen (1947) showed that angina brought on by exertion results in pain referred to a prior blister injury of the right elbow or the right mammary region, or to the site of an injury produced by injection of $5 \%$ saline into muscles of the back. In each of these cases, no angina pain was referred to these areas before the injuries, and the pain of the injuries had subsided prior to the angina attack which resulted in pain referred to these sites. Furthermore, Hutchins and Reynolds (1947) discovered that alterations in barometric pressure during high-altitude flights caused many of their patients to complain of pain localized to teeth which had been the site of previous painful stimulation (e.g., fillings, caries, and extractions), in many cases years earlier. Reynolds and Hutchins (1948) were able to replicate this finding under controlled conditions. One week after damaged teeth were filled or extracted, pinprick of the nasal mucosa produced pain referred to the previously treated teeth. This phenomenon occurred among patients who had been treated under general anesthesia, but not under the influence of a local anesthetic block. Furthermore, in patients who had bilateral dental treatment without a local anesthetic, subsequent blocks applied to one side permanently abolished the referred pain ipsilateral, but not contralateral, to the anesthetized side.

Theories which propose that central sensitization contributes to referred pain have also received recent support. Studies have shown a sensitization, or expansions of the receptive fields of dorsal horn neurons following inflammatory injury of various visceral tissues such as the urinary bladder (McMahon 1988), colon (Ness \& Gebhart 1990) and esophagus (Garrison et al. 1993), or following acute joint inflammation (Dougherty et al. 1992b; Schaible et al. 1987) or electrical stimulation of muscle afferents (Cook et al. 1987). Receptive field expansions have also been observed in trigeminal brainstem neurons following chemical stimulation of deep craniofacial afferents (Hu et al. 1992). Following inflammatory lesions of the rat knee joint, spinal dorsal horn (Neugebauer \& Schaible 1990) and thalamic 
(Guilbaud et al. 1986) neurons exhibit an enhanced responsiveness not only to mechanical stimulation of the inflamed joint, but also to stimulation of the muscles in the thigh and lower regions of both the ipsilateral and contralateral legs. These findings are consistent with clinical observations that hyperalgesia develops in body regions distant from a deep tissue injury (Hardy et al. 1950), and that flexion reflex thresholds are reduced in patients following gynecological surgery (Dahl et al. 1992a).

\subsection{Interactions of peripheral and central mechanisms in} referred pain. Perhaps the most controversial and interesting phenomena associated with referred pain relate to the effects of local anesthesia of the area of reference. Some early reports demonstrated that injection of a local anesthetic agent into the referred area reduces or eliminates the referred pain (Lemaire 1926; Morley 1931; Weiss \& Davis 1928). Others reported that referred pain was unaffected by local anesthesia of the referred area (Lewis 1942; McClelland \& Goodell 1943; Woollard et al. 1932). It may be that the equivocal findings in this area depend to a large extent on the intensity of the inputs from the injury. For example, Theobald (1941) showed that weak faradic stimulation of the cervix produced mild referred pain in the abdominal wall that was eliminated by local anesthesia of the referred area. Alternatively, intense stimulation produced referred pain which was unaffected by local anesthesia of the referred area. In support of this, Doran and Ratcliffe (1954) demonstrated that referred pain in the shoulder after phrenic nerve stimulation could be eliminated by local anesthesia of the referred zone. However, the effect of the anesthetic blockade could be counteracted by increasing the intensity of the stimulation. In addition, Bonica (1967) reported that referred pains of mild intensity associated with the early first stage of labour are virtually eliminated by local anesthesia of the lower abdominal wall, while referred pains of much greater intensity during the late first stage of labour are not affected by local anesthesia of the referred zone. Cohen (1947) has also described patients with left arm amputation who developed pain referred to the phantom arm during an attack of angina. In one patient, local anesthetic block of the brachial plexus eliminated the pain of angina referred to the phantom arm. In a second patient, pain in the phantom arm was reliably brought on by physical exercise. Before local anesthesia of the brachial plexus, pain in the phantom arm developed after the patient walked 120 to 150 yards. After a brachial plexus block the patient could walk up to 600 yards before pain re-appeared in the phantom arm.

The fact that referred pain can sometimes be reduced or eliminated by local anesthesia of the referred zone suggests that referred pain depends on a convergence of visceral and somatic inputs in the spinal cord. However, that local anesthesia is not effective when the visceral stimulus is very intense suggests that when visceral inputs are strong enough, a state of central sensitization develops, over-riding any requirement of input from the referred zone for maintenance of the pain. Thus, as in the case of secondary hyperalgesia, there appears to be an interaction between peripheral and central neural mechanisms underlying referred pain, and in some, but not all, cases afferent input is necessary for its maintenance. Using the terms we previously generated for the model of secondary hyperalgesia in Figure 2, we would argue that referred pain relies predomi- nantly on ongoing central sensitization, but that the net output of dorsal horn neurons which results in pain experience is also influenced by inputs from the referred area. If the tissue injury in the visceral organs is extensive enough to produce intense ongoing central sensitization, then inputs from the referred area are not required to produce referred pain. With less extensive injury, there is less ongoing central sensitization and local anesthesia of the referred area will alleviate the referred pain. The interdependence of referred and visceral pain on both the ongoing sensitization from the injured region and the inputs from the referred area are further exemplified by observations that patients with angina can be induced to suffer an angina attack not only by stressing the heart with exertion, but also by irritating the area of reference by producing ischemia in the left arm with a tourniquet (Cohen 1947). The influence of initial central sensitization on referred pain is indicated in cases where pain is referred specifically to the site of a previous injury.

\section{Neuropathic pain}

Pain that occurs as a result of nerve injury is by far the most complex somatosensory phenomenon that we know. Theories of neuropathic pain are probably as numerous as the conditions that lead to them. Symptoms of neuropathic pain include spontaneous pain, paroxysmal pain (episodic, shock-like pain), hyperpathia (pain which is delayed and exaggerated), hyperalgesia and allodynia, as well as extensive secondary hyperalgesia and the development of referred pains and focus points (see Bennett 1994 or Devor 1994 for a recent review). In many instances certain symptoms are closely associated with specific nerve pathologies. However, the diversity of symptoms and pathological changes, and the variability in the relationship between symptoms and pathology across patients allow for considerable debate about the etiology of neuropathic pain.

4.1. Peripheral neural mechanisms. Peripheral factors that contribute to neuropathic pain include an abnormal sensitization of nociceptors (Cline et al. 1989; Culp et al. 1989; Ochoa 1986), the development of abnormal adrenergic sensitivity in nociceptors (J. N. Campbell et al. 1988b; Wallin et al. 1976), the development of ectopic activity in damaged nerves, or in dorsal root ganglion (DRG) cells of damaged nerves (Devor et al. 1994; Kajander et al. 1992; Xie et al. 1995), the formation of ephaptic connections in demyelinated axons ( Jänig 1988), and abnormal sensitivity after collateral sprouting of primary afferent neurons (Inbal et al. 1987), among others.

Particularly important in many neuropathic pains is the involvement of the sympathetic nervous system, either through development of abnormal sympathetic function (Hoffert et al. 1984) or through its effects on abnormally functioning afferent nerves ( J. N. Campbell et al. 1994). It is clear from animal data, that in certain instances following nerve injury either nociceptors (Hu \& Zhu 1989; Sato \& Perl 1991), regenerating fibers within a neuroma (Wall \& Gutnik 1974; Devor \& Jänig 1981), or the somata of injured nerves (Devor et al. 1994; Kajander et al. 1992; Xie et al. 1995) develop an abnormal adrenergic sensitivity. It has also been observed following nerve injury in rats that rapidly adapting mechanoreceptors are modified such that 
they respond with low and irregular static discharges during a maintained mechanical stimulus ( $\mathrm{Na}$ et al. 1993). Importantly, these modified responses appear to depend on sympathetic efferent function, since they are blocked by intravenous administration of the adrenergic antagonist phentolamine. This type of sympathetic-sensory coupling mechanism results in high levels of afferent input in response to post-ganglionic sympathetic output. Pain syndromes such as causalgia and reflex sympathetic dystrophy are often relieved by sympathetic ganglion blocks (Bonica 1979). The importance of sympathetic nervous system involvement in many neuropathic pains has lead to the development of a new taxonomy of neuropathic pain. Current thinking distinguishes between sympathetically maintained pain (SMP) and sympathetically independent pain (SIP) (J. N. Campbell et al. 1994; Roberts 1986). SMP is defined as "pain attributable to sympathetic efferent function in peripheral tissues" (Campbell et al. 1994). SMP is, by definition, abolished when the sympathetic supply to the painful region is blocked. In contrast, SIP is not dependent upon sympathetic efferent function, so that maneuvers that are directed at blocking peripheral sympathetic activity do not affect the pain. One of the major advances of this taxonomy is to dissociate the presence of pain from signs of sympathetic disregulation (e.g., altered temperature, excessive sweating, trophic changes), so that evidence of abnormal sympathetic activity need not accompany SMP.

It is important to recognize that persistent pain and hyperalgesia are not linked exclusively with a single source of peripheral pathology. In some cases, hyperalgesia associated with nerve injury is alleviated by A-fiber nerve blocks (Meyer et al. 1985; Ochoa 1982; Wallin et al. 1976), while in other cases it is resistant to A-fiber block and is likely influenced by C-fibers (Ochoa \& Torebjörk 1989). In some instances, hyperalgesia associated with nerve injury is alleviated by sympathetic blocks, while in other cases it is either not relieved or even exacerbated by sympathetic blocks (Bonica 1979; Ochoa \& Marchettini 1993). Ochoa and Marchettini (1993) also point out that there are subsets of neuropathic pain patients which can be divided into "hot" and "cold" patients, based on the temperature of their limbs and the effects of temperature on their sensory symptoms. In "hot" patients, the affected skin is hot and pain is elicited by warming, while cooling provides relief of pain and hyperalgesia. In "cold" patients, the affected skin is cold, and pain is provoked by cooling, and may be relieved by warming.

\subsection{Central neural mechanisms in neuropathic pain. As} pointed out earlier, the development of post-injury adrenergic sensitivity in nociceptors and damaged nerves suggests that SMP can be explained entirely by peripheral mechanisms. However, several investigators have proposed that there is an interaction between peripheral and central neural factors which underlies SMP. Thus, Roberts (1986), who coined the term SMP, suggested that after peripheral nerve injury, an abnormal central state develops in which activity in sympathetic efferents stimulates low-threshold mechanoreceptors, which in turn induce pain by activating sensitized dorsal horn neurons. A similar type of interactive peripheral/central model for SMP has also been proposed by J. N. Campbell et al. (1994). According to their model, following injury, spontaneous pain results from asympathetic- sensory coupling mechanism in which nociceptors upregulate alpha-adrenergic receptors and respond to noradrenaline released from sympathetic terminals in the affected region. Sympathetically generated nociceptor activity produces a dynamically maintained state of central sensitization so that activity in low-threshold mechanoreceptors, which normally is not painful, now evokes allodynia in response to light touch. Blocking the sympathetic supply to the injured region interrupts the sympathetic-sensory coupling mechanism and restores the central neurons to a desensitized state, thus relieving both ongoing pain and allodynia.

Models such as these that propose an interactive role of sympathetic-sensory coupling in the periphery and central neural changes are extensions of an early proposal by Livingston (1943). Livingston argued that afferent activity generated from a peripheral nerve injury elicits an abnormal firing pattern within the spinal cord. A disturbance ensued in an internuncial pool of dorsal horn interneurons which resulted in reverberatory activity that eventually spread to other parts of the spinal cord. The spread of activity to the lateral spinal dorsal horns would have the effect of increasing sympathetic efferent activity, causing a disruption in vasoregulation, trophic changes, and hypersensitivity of peripheral tissue. The resultant increased sensory input, driven by sympathetic outflow, acts to maintain the abnormal firing in the spinal cord, creating Livingston's "vicious circle" of peripheral-central activity.

Recent animal data support the notion that nerve injury produces changes in central neural function. Nerve section leads to the development of increased neuronal activity at various levels of the somatosensory system. In addition to spontaneous activity generated from the neuroma (Wall \& Gutnik 1974), peripheral neurectomy also leads to increased spontaneous activity in the DRG (Burchiel 1984; Wall \& Devor 1983), dorsal spinal roots (Howe et al. 1977; Wiesenfeld \& Lindblom 1980), and spinal cord (Asada et al. 1990; David \& Aguayo 1980). Furthermore, after dorsal rhizotomy, there are increases in spontaneous neural activity in the dorsal horn (Basbaum \& Wall 1976; Loeser \& Ward 1967), the spinal trigeminal nucleus (Anderson et al. 1971; Macon 1979) and the thalamus (Albe-Fessard \& Lombard 1983; Lombard et al. 1979). There is also a lowered threshold for evoked activity in thalamic (Guilbaud et al. 1990) and cortical (Guildbaud et al. 1992a) neurons of rats with chronic constriction injuries of the sciatic nerve. These findings parallel those reported in the clinical literature in which patients with neuropathic pain after deafferenting lesions exhibit increased spontaneous neural activity in the somatosensory thalamus (Gorecki et al. 1989; Hirayama et al. 1989). Nerve section also produces expansions of the receptive fields of the neurons adjacent to those which are denervated by peripheral nerve sections (Devor $\&$ Wall 1978). These receptive field expansions have significant implications for the development of persistent pain after nerve sections. Thus, Markus et al. (1984) have demonstrated that the development of hypersensitivity in a rat's hindpaw following sciatic nerve section occurs concurrently with the expansion of the saphenous nerve's somatotopic projection in the spinal cord.

It is possible that receptive field expansions and spontaneous activity generated in the CNS following peripheral nerve injury are, in part, mediated by alterations in normal inhibitory processes in the dorsal horn. After peripheral 
nerve section, there is a reduction in the dorsal root potential, and the presynaptic inhibition it is assumed to represent (Wall \& Devor 1981). Nerve section also induces a reduction in the inhibitory effect of A-fiber stimulation on activity in dorsal horn neurons (Woolf \& Wall 1982). Furthermore, nerve injury affects descending inhibitory controls from brainstem nuclei. In the intact nervous system, stimulation of the locus coeruleus (Segal \& Sandberg 1977) or the nucleus raphe magnus (Oliveras et al. 1979) produces an inhibition of dorsal horn neurons. Following dorsal rhizotomy, however, stimulation of these areas produces excitation, rather than inhibition, in half the cells studied (Hodge et al. 1983).

Further evidence that loss of inhibitory control mechanisms contributes to pathological processing after nerve injury is indicated by the development of transynaptic degenerative changes in small and medium size neurons in lamina I-III of the spinal cord dorsal horn (Sugimoto et al. 1990). It has been suggested that the degeneration of neurons is triggered by intense stimulation associated with ectopic discharges in damaged nerves, and that the affected cells include inhibitory interneurons (Kajander \& Bennett 1992; Sugimoto et al. 1990). Importantly, both the degenerative changes (Sugimoto et al. 1990) and behavioral indices of enhanced pain sensitivity in animals (Yamamoto \& Yaksh 1993) are accentuated by intrathecal administration of strychnine, which, as a glycine antagonist, blocks inhibitory postsynaptic potentials.

Recently, several animal models of peripheral neuropathy have been developed which produce behavioral signs of hyperalgesia, allodynia, and spontaneous pain or dysesthesia. These behavioral signs bear a striking resemblance to symptoms of nerve injury-related pain in humans. These models involve placing either loosely constrictive ligatures around the entire rat sciatic nerve (Bennett \& Xie 1988), tight ligatures around $1 / 3$ to $1 / 2$ the rat sciatic nerve (Seltzer et al. 1990), or tight ligatures around the L5 and L6 spinal dorsal nerves (Kim \& Chung 1992), and observing the behavioral symptoms associated with the nerve pathology that develops over the next several days or weeks. Behavioral symptoms include hyperalgesia to radiant heat or mechanical stimulation, allodynia in response to warm or cold temperature stimulation, guarding of the affected limb (Attal et al. 1990; Bennett \& Xie 1988; Kim \& Chung 1992; Seltzer et al. 1990), and extraterritorial hyperalgesia and allodynia in adjacent uninjured tissue supplied by the saphenous nerve (Tal \& Bennett 1994). In addition to these behavioral signs, recent evidence suggests that these nerve constriction injuries produces profound changes in spinal cord physiology, including transynaptic degeneration (Sugimoto et al. 1990), increases in c-fos expression (Kajander et al. 1990) and the growth associated protein GAP 43 (Cameron et al. 1991), as well as decreases in tachykinin immunoreactive staining (Bennett et al. 1989; Cameron et al. 1991) in the dorsal horn. They also produce increased spontaneous activity and increased excitability (lowered thresholds to mechanical stimulation, and afterdischarges to suprathreshold stimuli) of spinothalamic tract cells (Palecek et al. 1992), as well as spontaneous discharges (Kajander et al. 1992; Xie et al. 1995) and increases in immunoreactivity of tyrosine hydroxylase (Chung et al. 1993) and nitric oxide synthase (Steel et al. 1994) in the DRG cells. Furthermore, the constriction injury leads to a dramatic increase in spinal cord metabolic (2-DG) activity in both the ipsilateral and contralateral spinal cord (Mao et al. 1992a). Since metabolic activity is increased in the absence of additional peripheral stimulation, it has been argued that the behavioral symptoms are driven by sustained alterations in spinal cord function. This notion is supported by the finding that there is a reduction in the hyperalgesia that develops following constriction injury of the sciatic nerve if the nerve is locally anesthetized at the time of injury (Dougherty et al. 1992a). Finally, while hyperalgesia and spontaneous pain produced by nerve constriction are reduced by post-injury local anesthesia of the sciatic nerve (Mao et al. 1992b) or sympathectomy (Kim et al. 1993; Shir $\&$ Seltzer 1991) suggesting a peripheral contribution to the pain pathology, they are also reduced by systemic or intrathecal administration of NMDA antagonists (Davar et al. 1991; Mao et al. 1992b), suggesting a central contribution as well.

4.3. Focal points and the maintenance of central sensitization. Associated with neuropathic pain is the development of focal points, which when stimulated produce painful sensations that are referred to a remote area. Livingston (1943) reported that pain, allodynia, and hyperalgesia associated with nerve injury can sometimes be alleviated by injections of local anesthetic into a focal point. Recently, Gracely et al. (1992) has presented experimental data from four patients who demonstrated a focus of unusually great cutaneous sensitivity, as well as allodynia and hyperalgesia in a remote region, at a distance from the focus. Stimulation in the focus produced intense pain in the allodynic and hyperalgesic skin. Local anesthesia of the focus eliminated the ongoing pain as well as the allodynia and hyperalgesia. They proposed a model of neuropathic pain in which ongoing nociceptive input from the focus maintains altered central processing that accounts for various sensory and motor abnormalities. Similar to the model we have discussed thus far in relation to cutaneous hyperalgesia after tissue injury and referred pain, the Gracely model relies on an interaction between alterations in central processing produced by an initiating stimulus and maintaining inputs from an ongoing peripheral source. According to Gracely et al. (1992), the original nerve injury alone, or in combination with noxious inputs due to subsequent surgical procedures, produces central neural changes, that are dynamically maintained for prolonged periods by ongoing input from peripheral pathological sources. Importantly, they suggest that altered central processing is a normal process, and not a pathology, but is maintained by pathological peripheral inputs. Other models of the central consequences of peripheral injury have also been proposed. A 1991 consensus statement highlights the role of a variety of peripheral triggers in inducing and maintaining central sensitization (Devor et al. 1991). Furthermore, recent data from Koltzenburg et al. (1994) indicates that the degree of allodynia or brush-evoked pain in patients with neuralgia is closely correlated with the degree of ongoing pain present in the affected limb. They also found that brush-evoked pain was prevented when ongoing pain in the affected limb was relieved by a regional guanethidine block or by local anesthetic blocks of nerves supplying the symptomatic skin.

Ongoing inputs which maintain the altered central processing can arise from any number of peripheral sources of the kind we have discussed previously. Since a specific 
peripheral source is not defined within Gracely's model, then similar neuropathic pains can be experienced by patients with very different peripheral pathologies. Thus, in SMP, activity in sympathetic efferents would contribute to the peripheral source, and in SIP, there would be another peripheral source (e.g., ephaptic connections, collateral sprouting, etc.). This may explain why patients with SMP and SIP often present with similar symptoms, and may also explain why patients on occasion have only some symptoms relieved by sympathetic blocks. According to Gracely et al., the key underlying mechanism is the altered central processing which can be maintained entirely, partially or not at all by abnormal peripheral sensitivity to sympathetic efferent activity.

Another similarity between Gracely et al.'s model and that presented in Figure 2 is the reliance of altered central processing on inputs associated with the test stimuli. Thus, Gracely described a case where repeated stimulation of the focus area resulted in reports of increased spontaneous pain and allodynia from the remote area. The altered central processing depends not only on an initial injury for its development and on ongoing peripheral inputs for its maintenance, but also on the magnitude of the peripheral inputs for its expression. They also argued that since pain, allodynia, hyperalgesia, and the sensitivity of the focus reappeared after local anesthesia of the focus area wore off, the altered central processing persists in a silent state until reactivated by the peripheral source. This raises the possibility that long-term blocking of the peripheral source may be necessary to reverse the altered central processing, and could produce a prolonged pain free period that outlasts the peripheral block. Evidence for this comes from clinical reports that a series of anesthetic or peripheral sympathetic blocks sometimes produces long-lasting pain relief in patients with neuropathic pain (Benedetti 1993; Bonica 1979; Livingston 1943).

Using our own terms, Gracely et al.'s hypothesis could be redefined as being reliant on both initial central sensitization and ongoing central sensitization. The two components contribute to net central sensitization, along with additional peripheral inputs from test stimuli or other sensory stimuli which contribute to the net activity of dorsal horn cells underlying neuropathic pain. Although there are many similarities between Gracely et al.'s model and our own, the main difference is their proposal that the altered central processing depends critically on maintaining inputs from peripheral sources (although they do propose that with prolonged peripheral input the altered central processing may become autonomous of the peripheral input). In contrast, we propose that if the initial central sensitization is great enough, then the net central sensitization will allow pain and hyperalgesia to persist in the absence of ongoing central sensitization from a peripheral source. Thus, secondary stroking hyperalgesia after faradic skin stimulation, or hyperalgesia to punctuate stimuli after capsaicin injection, will persist after local anesthesia of the injured skin.

\subsection{Pain in phantom limbs and deafferented structures.} Mitchell (1872) coined the term "phantom limb" to describe the well know phenomena in which amputees continue to report a sensory awareness of a limb that has been amputated. The term phantom limb pain (PLP) is now commonly used to describe pains which are referred to the phantom limb. The idea that pain can be referred to a phantom limb clearly implies that central neural mechanisms are involved. Unlike the peripheral theories of referred pain, there is absolutely no opportunity for PLP to result from a branching of nerves from an injured region to the area of reference. The simplest hypothesis is that inputs from damaged nerve trunks, stump neuromas, or DRG cells generate signals which are transmitted to the CNS where they are interpreted as coming from the amputated limb, so that pain is projected to that region. This explanation is similar to the convergence-projection hypothesis of referred pain, in that pain is projected to the amputated limb because of a misinterpretation of the origin of the input; however, with PLP there is no need to propose converging inputs, since the transected nerves in the stump continue to innervate the same spinal cord cells after amputation.

Some of the original descriptions of Mitchell (1872), as well as an accumulating body of more recent evidence, suggests that PLP depends not only on a mislocation of the origin of input, but also a sensitization of central neurons prior to, or during, amputation. A striking property of PLP is the persistence of a pain that existed in a limb prior to its amputation (Melzack 1971). Case studies of amputees (see Katz \& Melzack 1990) have demonstrated pain "memories" of painful diabetic and decubitus ulcers, gangrene, corns, blisters, ingrown toenails, cuts, and deep tissue injury. In addition, the phantom limb may assume the same painful posture as that of the real limb prior to amputation (Katz \& Melzack 1990). Mitchell described this phenomena in one of his patients: "Another class has the hand constantly in some painful position which it occupied before the operation, so that the last real sensation is so stamped upon the sensorium as to forbid its erasure by any future impression."

It has been reported that as many as 79\% of amputees describe their phantom pains as similar to pains felt in the limb before amputation (Katz \& Melzack 1990). Reports of pain memories in phantom limbs appear to be less common when there has been a discontinuity, or a pain-free interval, between the experience of pain and the amputation. This may explain why relief of preamputation pain by continuous epidural block for 3 days prior to amputation (Bach et al. 1988), as well as after amputation (Jahangiri et al. 1994), decreases the incidence of PLP 6 months later. Furthermore, there appears to be a higher probability that pain will persist in the phantom limb if pain is experienced at or near the time of amputation ( Jensen et al. 1985; Katz \& Melzack $1990)$, or if preamputation pain is very intense or of long duration (Jensen et al. 1985; Jensen \& Rasmussen 1994).

There is also a literature on the persistence of painful and nonpainful sensations associated with removal or deafferentation of body structures other than the limbs, including breasts (Kroner et al. 1989), teeth (Hutchins \& Reynolds 1947; Reynolds \& Hutchins 1948), and internal and special sense organs. Ulcer pain has been reported to persist after vagotomy (Szasz 1949) or subtotal gastrectomy with removal of the ulcer (Gloyne 1954). Similarly, patients have reported labor pain and menstrual cramps following total hysterectomy (Dorpat 1971), rectal and hemorrhoid pain following removal of the rectum (Ovensen et al. 1991), the burning pain of cystitis after complete removal of the bladder (Brena \& Sammons 1979), and the pain of a severely ulcerated cornea after enucleation of an eye (Minski 1943). 
Pain also persists in patients with deafferentation that does not involve amputation. Patients with brachial plexus avulsions (Jensen \& Rasmussen 1994; Reisner 1981) and spinal cord injuries often experience pain in the anesthetic, deafferented region (Berger \& Gerstenbrand 1981; Conomy 1973). For example, Nathan (1962) described a patient who continued to feel the pain of an ingrown toenail after a complete spinal cord break. In addition, patients with injuries of the brachial plexus (Jensen \& Rasmussen 1994; Reisner 1981) or spinal cord (Berger \& Gerstenbrand 1981; Conomy 1973; ) sometimes report that a limb is in the same uncomfortable, and often painful, posture it was in prior to the injury or block.

PLP or deafferentation pain is not entirely independent of peripheral inputs. In some instances there is a reactivation of pain experienced before amputation that is brought on by peripheral stimulation. Leriche $(1947 \mathrm{a} ; 1947 \mathrm{~b}) \mathrm{de-}$ scribed a patient who did not experience PLP until 6 years after amputation, when an injection into the stump instantly, and permanently, revived the pain of a former painful ulceration of the Achilles tendon. Nathan (1962; 1985) reported a similar phenomenon when applying noxious stimulation to the stump of an amputee who later reexperienced the pain of an ice-skating injury he had sustained 5 years earlier when the leg was intact. Noordenbos and Wall (1981) also described 7 patients with partial peripheral nerve injury and subsequent pain, who underwent complete nerve resection and graft or ligation. Following regeneration and a pain-free period, all redeveloped pain of the same quality and in the same location as the pain they had experienced prior to nerve resection, although in some patients the recurrence of pain was restricted to a smaller area within the originally painful region. These studies and case reports indicate that previous pains may be reactivated months or even years after the original injury, in some cases by a peripheral trigger which provides the input required to activate the sensitized central neurons. In the case of amputation phantoms, likely candidates for peripheral triggers include ectopic output from neuromas and DRGs.

4.5. Phantom-like pain in animals. Deafferentation by peripheral neurectomy or dorsal rhizotomy in rodents is followed by self-mutilation (autotomy) in which the animals bite and scratch the insensate paw to the point of amputation (Wall et al. 1979). There is evidence that autotomy behavior is produced by ongoing pain or dysesthesia, associated with increased neuronal activity, which is referred to the anesthetic region (Blumenkopf \& Lipman 1991; Coderre et al. 1986; however, also see Rodin \& Kruger 1984; Sweet 1981). Autotomy behavior is dramatically affected by alterations in the level of noxious input present at the time of, or prior to, nerve section. Thus, noxious chemical (Coderre et al. 1986; Dennis \& Melzack 1979), thermal (Coderre \& Melzack 1985; 1987; Katz et al. 1991), and electrical (Katz et al. 1991; Seltzer et al. 1991) stimulation prior to nerve sections significantly increases the severity of autotomy following neurectomy or rhizotomy. These findings suggest that the prior injury produces central changes which influence nociceptive behavior, after nerve sections, at a time when inputs from the injured region are no longer capable of transmitting their message centrally. In contrast to the effect of increasing noxious inputs at the time of nerve injury, reducing or eliminating the afferent barrage induced by nerve section produces a dramatic reduction in autotomy. When the afferent barrage induced by nerve cuts in rats is blocked by treating the sciatic and saphenous nerves with local anesthetics prior to sectioning them, there is a significant reduction in the incidence and severity of autotomy (González-Darder et al. 1986; Seltzer et al. 1991). It has also been shown that intrathecal treatment with morphine $1 \mathrm{hr}$ before, but not $15 \mathrm{~min}$ after sciatic nerve section, resulted in a significant reduction in the severity of autotomy lasting for least 28 days (Puke \& WeisenfeldHallin 1993).

Katz et al. (1991) recently developed an animal model which parallels the observation that human amputees report similar pains in a limb before and after amputation. In this animal model, rats selectively initiated autotomy in either the lateral or medial half of a hindpaw if that particular half had been given a thermal injury prior to sciatic and saphenous nerve sections. The selective attack on the previously injured region, despite the fact that the entire foot was deafferented, suggests that the rats were responding to pain referred to the injured area, which was associated with the prior injury and the central sensitization it produced. Rats injured after neurectomy did not show a similar preference indicating that the rats were not responding simply to peripheral cues associated with the injury.

4.6. Role of ongoing inputs in phantom limb pain (PLP). Both the human and animal data suggest that the initial central sensitization is critical to the development of PLP or pain in deafferented structures. However, as described previously, PLPs which resemble pre-amputation pain sometimes require a peripheral stimulus to trigger their onset. In other cases, PLP can be relieved by local anesthesia of focal points in the stump (Livingston 1943). Thus, while PLP may be initiated by central sensitization associated with an injury barrage, there is a role for peripheral inputs and potentially ongoing central sensitization for the maintenance of PLP. In many cases, PLPs resolve themselves within a few months of amputation. It is expected that in these cases PLP is originally driven primarily by inputs from ectopic activity in stump neuromas or DRG cells acting on dorsal horn neurons sensitized by the preamputation injuries and/or the injury barrage associated with the nerve sections at the time of amputation. The PLP subsides when the damaged nerves heal adequately, minimizing ongoing inputs from the stump neuromas or DRG cells. PLP may continue if the initial sensitization is of sufficient intensity, if peripheral tissues do not heal adequately, or if stump neuromas or DRG cells develop a sensitivity to sympathetic efferent activity so that ongoing peripheral inputs produce an ongoing sensitization. Furthermore, the fact that PLP can be triggered months or years after the amputation suggests that initial central sensitization produces a lasting influence on central processing, which can reinstate a painful condition if appropriate dorsal horn neurons and/or more rostral sensory structures are activated by peripheral triggering inputs.

\section{Postoperative pain}

Early this century, Crile (1913) first proposed that CNS changes produced by tissue damage and noxious inputs associated with surgery could contribute to postoperative 
pain. However, it was only after the recent finding of Woolf and Wall (1986) provided a sound justification for preemptive treatment, that this idea began to receive the clinical attention it deserves. Woolf and Wall (1986) demonstrated in experimental animals that opioids are much more effective at reducing stimulus-induced increases in the excitability of the dorsal horn if they are administered prior to, rather than following, $\mathrm{C}$-fiber electrical nerve stimulation. Recent clinical evidence supports the hypothesis that the administration of analgesic agents prior to surgery may prevent the central sensitizing effects of the surgical procedure. Thus, it may be possible to reduce postoperative pain intensity or lower post-operative analgesic requirements for periods much longer than the duration of action of the preoperatively administered agents.

5.1. Pre-emptive analgesia. A growing body of clinical data shows that preoperative local (Jebeles et al. 1991; Rademaker et al. 1991; Ringrose et al. 1984; Tuffin et al. 1989; Tverskoy et al. 1990) or spinal (Bugedo et al. 1990; Heard et al. 1992; Tverskoy et al. 1990) anesthesia, or the epidural preadministration of analgesic agents (Campbell et al. 1990; Kiss \& Killan 1992; McQuay et al. 1988; Richmond et al. 1993), can significantly reduce postoperative pain or postoperative opioid requirements (see Woolf \& Chong 1993 for review). The analgesic effects of such preoperative treatments are assumed to depend on the ability of the pretreatment to preempt the surgically induced sensitization of central nervous system (CNS) neurons; the term preemptive analgesia has been coined for such treatments (Wall 1988). Although there is considerable evidence to show that peripheral injury, as would occur with surgery, leads to a sensitization of CNS neurons (Hylden et al. 1989; Kenshalo et al. 1979; McMahon \& Wall 1984; Perl 1976; Simone et al. 1991; Woolf \& King 1990), the evidence for preemptive treatments to attenuate postoperative pain to a clinically significant degree is less convincing (Dahl 1994; Katz 1995; McQuay 1995). The conclusiveness of the evidence is hampered by the failure to adequately address whether the same treatment started after surgery could produce the same therapeutic benefit. Initial studies examined the effects of preemptive treatments on postoperative pain as compared with no treatment. Although studies of pretreatment versus no treatment were overwhelmingly suggestive of a beneficial effect of preemptive analgesia, its value became less obvious when compared with the same treatment initiated after surgery (i.e., pre- vs, postsurgery). Studies comparing the effectiveness of pre- versus postsurgical treatment using local anesthetic infiltrations, systemic analgesia or regional administration of opioids or local anesthetic agents have produced equivocal results, with some studies indicating a small beneficial effect (Ejlersen et al. 1992; Katz et al. 1992a; 1994; Richmond et al. 1993) and others no effect (Dahl et al. 1992b; Dierking et al. 1992; Pryle et al. 1993; Rice et al. 1990).

One explanation for the lack of clinically significant benefits of presurgical administration of opioids or local anesthetic agents has been that in some clinical trials pre- or intraoperative opioids are used routinely as part of the general anesthetic regimen in both pre- and postsurgical treatment groups (Katz et al. 1992b). Thus, it is possible that the pre/intraoperative opioid use may confound the results, since they may themselves produce a preemptive effect that reduces postoperative pain (Katz et al. 1996;
Yashpal et al. 1996). Another explanation is that in some instances postoperative pain may depend more heavily on the peripheral inflammation that follows surgery than on central sensitization that occurs during surgery (Coderre et al. 1993; Woolf \& Chong 1993), and consequently postsurgical treatments may be as effective as presurgical treatments.

There is now recent evidence that the preemptive effect is mediated by the NMDA receptor-ion channel complex (Roytblat et al. 1993; Tverskoy et al. 1994) since patients administered intraoperative ketamine (a clinically available anesthetic with NMDA channel blocking properties) but not a placebo show a reduction in postoperative mechanical hyperalgesia at the incision site two days after surgery (Tverskoy et al. 1994), and significantly reduced morphine requirements during the first $24 \mathrm{hrs}$ after surgery (Roytblat et al. 1993). In both these studies, the preemptive effects of ketamine were observed at least $24 \mathrm{hrs}$ after the duration of ketamine's pharmacological action.

5.2. Animal models of pre-emptive analgesia. In the animal literature, the formalin test has been used as a model of injury-induced central sensitization (Coderre et al. 1990), and as a model for studying the mechanisms underlying preemptive analgesia (Abram \& Yaksh 1993; 1994; Goto et al. 1994). Subcutaneous injection of dilute formalin into a rat's paw produces a biphasic response including an early intense response in the first $5 \mathrm{~min}$, and a later moderate response that is expressed from 20 to $60 \mathrm{~min}$ after injection (Dubuisson \& Dennis 1977). The nociceptive response to subcutaneous formalin injection is matched by a corresponding biphasic increase in the activity of dorsal horn neurons after such injection (Dickenson \& Sullivan 1987a). It has been demonstrated that intrathecal (i.t.) administration of either lidocaine (Abram \& Yaksh 1994; Coderre et al. 1990) or opiates (Abram \& Yaksh 1993; Dickenson \& Sullivan 1987a) abolishes behavioral and dorsal horn neuron responses to subcutaneous formalin, if they are administered prior to, but not immediately after, the early phase of the formalin response. This suggests that neural activity generated during the early phase of the formalin response is capable of producing changes in CNS function which in turn influence nociceptive processing during the late phase. The ability of the preinjury treatment with i.t. lidocaine or opiates to suppress the late phase response to formalin has been described as an animal model of preemptive analgesia, since the pretreatments are able to preempt the central sensitization which contributes to persistent postinjury nociceptive behaviors.

Recently, we have demonstrated that the ability of i.t. lidocaine to preempt postinjury nociception in the formalin test was lost as the concentration of formalin was increased from 2.5 to $5 \%$ (Yasphal et al. 1996). A strong preemptive effect (i.e., a significant reduction in nociceptive scores) of lidocaine was obtained in rats given $2.5 \%$ formalin. This preemptive effect was reduced (resulting in significantly higher nociceptive scores) in a concentration-dependent manner in rats given 3.75 and $5 \%$ formalin. In the same study, we found that while a significant and concentrationrelated degree of inflammation (plasma extravasation) was produced by 3.75 and $5 \%$ formalin, the degree of inflammation produced by $2.5 \%$ formalin was not significantly different than that produced by the same volume $(50 \mu \mathrm{l})$ of saline, and was only slightly, but not significantly higher 
than no injection at all (Yasphal et al. 1996). Thus, the preemptive effects of i.t. lidocaine were greatest when there was little or no inflammation, and decreased directly with increases in peripheral inflammation. One implication of these findings is that it may be difficult to demonstrate a significant effect of preemptive analgesia after surgical procedures which produce extensive peripheral injury, accompanied by considerable local inflammatory changes. In this manner, the peripheral inflammatory changes and afferent input associated with postoperative inflammation may over-ride much of the beneficial effect of blocking afferent inputs at the time of surgery (but also see Katz et al. 1994). A second implication of this finding is that it is important to pay careful attention to the concentration of formalin that the investigators have used, when comparing the results of different studies using the formalin test. This distinction may explain the recent finding that there is no difference between pre- and posttreatment with i.t. lidocaine or excitatory amino acid antagonists (Chapman \& Dickenson 1993) or opioids (Chapman et al. 1994) on the dorsal horn neuronal responses to a peripheral injection of $5.0 \%$ formalin to rats' toes, and why posttreatment with the NMDA antagonist AP5 produced a significant reduction in nociceptive responses to hindpaw injection of $10 \%$ formalin in mice (Murray et al. 1991). Furthermore, it may explain why late phase dorsal horn neuronal responses to $5.0 \%$ formalin are significantly reduced by local anesthesia of the injected area at the time of testing (Dickenson \& Sullivan $1987 \mathrm{~b}$ ), but not by a prior local anesthesia of the injected area during the early phase (Haley et al. 1990).

\subsection{Preemptive analgesia and models of persistent pain} in animals. In addition to providing experimental evidence for a possible explanation as to why preemptive treatments may not always be effective for reducing of postoperative pain, our results may also explain why pretreatments are not always more effective than posttreatments for the alleviation of nociception after peripheral tissue injury in animals. We have previously (Coderre 1993) pointed out, for example, that the effectiveness of pre- versus posttreatment with the N-methyl-D-aspartate receptor antagonist MK-801 as an antinociceptive agent is very much dependent on the type of nociceptive test that is used. In the case of peripheral neuropathy following nerve constriction injury in rats (Davar et al. 1991; Mao et al. 1992a; 1992b; Yamamoto \& Yaksh 1992a), it has been shown that MK-801 blocks or reduces hyperalgesia regardless of whether the drug administration is initiated prior to or following the injury. Furthermore, MK-801 effectively relieves hyperalgesia associated with carrageenan-induced inflammation when given as a posttreatment (Ren et al. 1992; Yamamoto et al. 1993). However, in the rat formalin pain model, NMDA antagonists have differential effects depending on whether they are administered pre- or postinjury. MK-801 significantly reduces nociceptive behaviors following formalin injury of a rat's hindpaw if administered prior to, but not following, the early phase response to formalin (Coderre \& Melzack 1992; Yamamoto \& Yaksh 1992b). Furthermore, the effects of pretreatment with MK-801 or other NMDA antagonists are much more pronounced on the late phase responses to formalin, than on early phase responses, either on behavioral measures of nociception (Coderre \& Melzack 1992; Millan \& Seguin 1993; Yamamoto \& Yaksh 1992b), or on electrophysiological measures of dorsal horn neuronal activity (Haley et al. 1990). We suggest that either pre- or posttreatments are effective in nociceptive tests involving neuropathy or significant peripheral inflammation where there is ongoing afferent input, whereas pretreatments are more effective than posttreatments in the case of low concentration formalin-induced nociception, which depends to a large extent on an initial afferent barrage and central sensitization that occurs at the time of formalin injection. However, as discussed in the previous section this assumption may break down as the concentration of formalin is increased.

5.4. Contribution of ongoing inputs to postoperative pain. It is expected that with cutaneous hyperalgesia, referred pain, and neuropathic pain, postoperative pain is influenced both by central sensitization associated with an injury barrage during surgery as well as by ongoing peripheral inflammatory inputs that contribute to central sensitization after surgery. Thus, the effects of preemptive analgesia are dependent not only on the intensity of the initial barrage, but also on the degree of peripheral inflammation that develops after surgery. Although preemptive effects may be expected to be best demonstrated in cases of extensive surgical trauma in which there is a strong initial central sensitization, these may also be the same cases where a postsurgery treatment will also be effective by alleviating ongoing central sensitization due to ongoing inputs from inflamed peripheral tissue (Katz et al. 1993; Woolf \& Chong 1993). It would be interesting to assess whether preemptive treatments would be most effective in minimizing postoperative pain for surgical procedures that result in damage to major nerves, thus producing a larger initial afferent barrage. Furthermore, it has recently (Katz et al. 1995) been demonstrated in a 2-year follow up of patients who had undergone lateral thoracotomy that the incidence of chronic post-thoracotomy chest wall pain was pronounced $(>60 \%)$, whether patients received preemptive analgesic or postincisional treatments. These results suggest that although preemptive treatments may reduce post-operative pain and analgesic consumption in the immediate postoperative period, these short-term beneficial effects have little or no bearing on the development of chronic postthoracotomy pain. It may be useful to extend preemptive treatment into the postoperative period to prolong the initial advantage conferred by the preoperative blockade in order to protect against long-term postoperative pain problems. Thus, the use of balanced analgesia (Dahl et al. 1990), with multiple agents and routes of administration to block nociceptive activity in the pre-, intra- and postoperative periods (e.g. Jahangiri et al. 1994), may be more useful from a long-term clinical perspective, than brief, preemptive treatments restricted to the pre- or intraoperative period.

\section{Conclusions}

We have examined clinical and experimental evidence in four key areas of pain research: cutaneous hyperalgesia, referred pain, neuropathic pain, and postoperative pain. In each there is evidence that persistent pain depends not only on central sensitization, but also on inputs from damaged peripheral tissue. Central sensitization may be comprised of both initial and ongoing components, each driven by variable levels of input from peripheral sources. Each of these factors - initial sensitization, ongoing central sensitiz- 